\title{
Quantum chaotic tunneling in graphene systems with electron-electron interactions
}

\author{
Lei Ying, ${ }^{1}$ Guanglei Wang, ${ }^{1}$ Liang Huang, ${ }^{1,2}$ and Ying-Cheng Lai ${ }^{1,3,}{ }^{*}$ \\ ${ }^{1}$ School of Electrical, Computer, and Energy Engineering, Arizona State University, Tempe, Arizona 85287, USA \\ ${ }^{2}$ Institute of Computational Physics and Complex Systems, and Key Laboratory for Magnetism and Magnetic Materials of MOE, \\ Lanzhou University, Lanzhou, Gansu 730000, China \\ ${ }^{3}$ Department of Physics, Arizona State University, Tempe, Arizona 85287, USA
}

(Received 1 April 2014; revised manuscript received 2 December 2014; published 16 December 2014)

\begin{abstract}
An outstanding and fundamental problem in contemporary physics is to include and probe the many-body effect in the study of relativistic quantum manifestations of classical chaos. We address this problem using graphene systems described by the Hubbard Hamiltonian in the setting of resonant tunneling. Such a system consists of two symmetric potential wells separated by a potential barrier, and the geometric shape of the whole domain can be chosen to generate integrable or chaotic dynamics in the classical limit. Employing a standard mean-field approach to calculating a large number of eigenenergies and eigenstates, we uncover a class of localized states with near-zero tunneling in the integrable systems. These states are not the edge states typically seen in graphene systems, and as such they are the consequence of many-body interactions. The physical origin of the non-edge-state type of localized states can be understood by the one-dimensional relativistic quantum tunneling dynamics through the solutions of the Dirac equation with appropriate boundary conditions. We demonstrate that, when the geometry of the system is modified to one with chaos, the localized states are effectively removed, implying that in realistic situations where many-body interactions are present, classical chaos is capable of facilitating greatly quantum tunneling. This result, besides its fundamental importance, can be useful for the development of nanoscale devices such as graphene-based resonant-tunneling diodes.
\end{abstract}

DOI: 10.1103/PhysRevB.90.224301

PACS number(s): 05.45.Mt, 03.65.Pm, 73.63.-b, 73.61.Wp

\section{INTRODUCTION}

Quantum chaos is referred to as the study of quantum manifestations of chaotic dynamics in the corresponding classical system [1,2], a field that has been active for more than three decades. In closed chaotic Hamiltonian systems, the basic phenomena that have been and continue to be studied include energy level-spacing statistics [3-5] and quantum scarring [6-32]. In open Hamiltonian systems, quantum chaotic scattering [33-43] has been investigated extensively. Quite recently, due to the significant development of graphene physics [44-50], relativistic quantum manifestations of classical chaos have become an interesting area of study [51-65]. However, in these works on quantum chaos, the standard setting was that of single-particle quantum dynamics, whereas many-body effects such as electron-electron interactions were ignored. While there were also previous studies of the interplay between many-body interactions and classical chaos [66-69], these were exclusively for nonrelativistic quantum systems described by the Schrödinger equation. To investigate the effect of chaos on relativistic quantum systems with manybody interactions has thus been an outstanding problem, yet it is not only fundamental to physics, but also important for the practical development of relativistic quantum devices.

To study quantum chaos in the presence of many-body interactions, in this paper we use the standard Hubbard model with on-site repulsive Coulomb interactions. This paradigmatic model to treat interacting particles in a lattice was originally proposed [70] to describe the transition between conducting and insulating systems. For electrons in a solid, comparing with the conventional tight-binding model representing a

\footnotetext{
*Ying-Cheng.Lai@asu.edu
}

single electron Hamiltonian, the Hubbard model contains a potential term to include the many-body effect through the mechanism of on-site Coulomb interaction [71,72]. There has been a great deal of interest in the Hubbard model due to its relevance to frontier problems in condensed matter physics such as high-temperature superconductivity and the trapping of untracold atoms in optical lattices [71]. As we demonstrate in this paper, while the Hubbard model is much more challenging and sophisticated than the tight-binding model, it can serve as a paradigm to gain significant physical insights into many-body relativistic quantum manifestations of distinct type of classical dynamics.

To be concrete, we focus on graphene systems and study the particular phenomenon of quantum resonant tunneling. The typical setting of a quantum tunneling system consists of two symmetric potential wells separated by a potential barrier in between, as shown schematically in Fig. 1. The whole system, which includes the left and right wells as well as the barrier, is closed, and its geometrical shape can be chosen to yield characteristically distinct types of dynamics in the classical limit. For example, if the whole system has a rectangular domain, the classical dynamics is integrable, but fully developed chaos can arise if the system has a stadium or a bowtie shape. It was discovered that, in both nonrelativistic [73] and relativistic [58] quantum, single-electron tunneling systems, classical chaos can regularize quantum tunneling dynamics. Here by "regularizing" we mean that the spread in the tunneling rate in any small energy interval, typically seen in the integrable geometry, can be greatly suppressed when the underlying geometry becomes chaotic. A unique feature in the relativistic case, as demonstrated in Ref. [58], is the high tunneling rate in the regime where the particle energy is smaller than the height of the potential barrier. This is a manifestation of the Klein-tunneling phenomenon. 
(a)

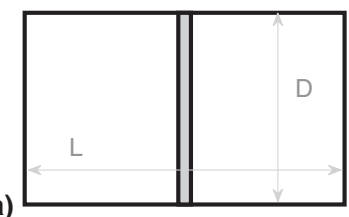

(c)

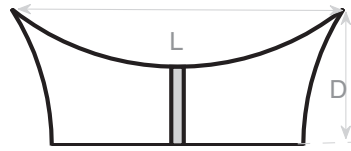

FIG. 1. Schematic illustration of four classes of geometrical domains for graphene billiards studied in this paper: (a) rectangle, (b) stadium, (c) bowtie, and (d) mushroom. The respective classical dynamics are integrable (a), chaotic with neutral periodic orbits (b), hyperbolic with all periodic orbits being unstable (c), and nonhyperbolic with mixed phase space (d). The thin gray region along a symmetric line represents the potential barrier.

In spite of the recent results on regularization of quantum tunneling by chaos $[58,73]$ in the single particle framework, whether the same can be achieved when many-body interactions are present was unknown prior to this work. More generally, the interplay among chaos, many-body interactions, and relativistic quantum mechanics is a fundamental yet outstanding issue that we aim to address here. There are two main findings: (1) emergence of a class of localized, spinpolarized, relativistic quantum states in classically integrable domains, which find no counterpart in the single-particle framework, and (2) effective removal of these states by classical chaos. A more detailed explanation of these findings is as follows.

In order to uncover the unique relativistic quantum phenomena caused by classical chaos in the presence of many-body interactions, we first study the class of integrable systems of rectangular shape [Fig. 1(a)]. Since the whole system is closed, we calculate the eigenenergies and investigate various eigenstates from the mean-field Hubbard Hamiltonian. A striking finding of this work is emergence of a class of eigenstates with near zero tunneling rate. In particular, for such an eigenstate, the spin-up and spin-down wave functions are typically separated, i.e., the spin-up electrons reside in only one potential well while the spin-down electrons reside in the other. As a result, if the initial state is spin-up in one potential well, it is localized and will stay in the same well practically for an infinite amount of time with little quantum tunneling. When the potential term characterizing the on-site Coulomb interactions is removed so that the Hamiltonian becomes that of the tight-binding type, such localized states no longer exist, indicating strongly that they are the result of electron-electron interactions and consequently a distinct many-body phenomenon. We derive an approximate theory, based on the simplified picture of one-dimensional tunneling of massless Dirac fermions, to explain the physical origin of the localized states. We further find that, when the geometrical shape is that of stadium [Fig. 1(b)] or bowtie [Fig. 1(c)] so that the classical dynamics is chaotic, the localized states are effectively removed and the tunneling rates become significant. This means that classical chaos is capable of destabilizing the localized states. In addition to the classically integrable and fully chaotic domains, we have also considered a class of domains, the mushroom billiard [Fig. 1(d)], in which the classical dynamics is mixed (or nonhyperbolic) with coexisting regular and chaotic components in the phase space $[65,74]$. We show that, due to the chaotic component, quantum tunneling can be regularized and enhanced. From the standpoint of device development such as graphene-based resonant-tunneling diodes, the localized states present an obstacle to effective tunneling and such states are therefore undesirable. From this perspective, classical chaos may be regarded as advantageous.

In Sec. II we describe the mean-field Hubbard model and our method to compute the tunneling rate and tunneling probability for graphene systems. In Sec. III we present evidence for polarized states with near-zero tunneling rate as induced by many-body interactions in classically integrable domains and demonstrate that chaos can regularize the quantum many-body tunneling dynamics. In Sec. IV we present a physical theory based on solutions of the Dirac equation to understand the emergence of polarized states. Conclusions and discussion are presented in Sec. V.

\section{METHODS: MEAN-FIELD HUBBARD MODEL AND QUANTUM TUNNELING}

\section{A. Mean-field Hubbard Hamiltonian}

We consider $p_{z}$ orbitals contributing to $\pi$-electron hopping in the graphene honeycomb lattice. Each orbital can have two electrons at most, one spin-up and another spin-down. To capture the essential physics of electron-electron interactions in graphene while keeping the model tractable, we take into account nearest-neighbor hopping terms and electron Coulomb repulsion at the local site. The tight-binding Hubbard Hamiltonian has the following standard form [75]:

$$
\begin{aligned}
H= & -t \sum_{\langle i, j\rangle, \sigma} c_{i, \sigma}^{\dagger} c_{j, \sigma}+V(x, y) \sum_{i, \sigma} c_{i, \sigma}^{\dagger} c_{i, \sigma} \\
& +U \sum_{i, \sigma} n_{i, \sigma} n_{i, \bar{\sigma}}
\end{aligned}
$$

where the summation of $\langle i, j\rangle$ is with respect to all nearestneighbor pairs, the index $\{\sigma, \bar{\sigma}\}$ denotes spin-up and spin-down electrons, $c_{i, \sigma}^{\dagger}\left(c_{j, \sigma}\right)$ is the creation (annihilation) operator, $n_{i \sigma}=c_{i, \sigma}^{\dagger} c_{i, \sigma}$ is the number operator, the nearest-neighbor hopping energy is $t=2.8 \mathrm{eV}, V(x, y)$ is the location dependent external electric potential, and $U$ is the Coulomb energy describing the interaction between a spin-up and a spin-down electron at the same site. While the Hubbard Hamiltonian provides a somewhat simplified picture of electron-electron interactions in the corresponding system, the analysis and computations become extremely difficult even for moderate system size with only tens of atoms. For relatively large system size, approximation must be employed to gain physical understanding of the system behaviors. A standard approach is to use the mean-field approximation, where the Hamiltonian (1) 
is modified to [76-81]

$$
\begin{aligned}
H_{\mathrm{MF}}= & -t \sum_{\langle i, j\rangle, \sigma} c_{i, \sigma}^{\dagger} c_{j, \sigma}+V(x, y) \sum_{i, \sigma} c_{i, \sigma}^{\dagger} c_{i, \sigma} \\
& +U \sum_{i, \sigma}\left\langle n_{i, \bar{\sigma}}\right\rangle n_{i, \sigma} .
\end{aligned}
$$

Physically the mean-field Hamiltonian describes the situation where a spin-up electron at site $i$ interacts with the average spin-down electron population $\left\langle n_{i \downarrow}\right\rangle$ at the same site, and vice versa. The mean-field Hubbard model is effectively a variation of the unrestricted Hartree-Fock approximation [82]. There have been recent efforts in comparing the various aspects of the mean-field Hubbard model with those from first-principle or quantum Monte Carlo calculations, with the conclusion that the mean-field approximation is generally valid for graphene systems [80,81], especially in the weakly coupling regime $[77,83]$. It is thus justified to choose the parameter $U$ below the critical Coulomb repulsion $U_{c}=2.2 t$. In this paper we use $U=1.2 t$ [81].

System (2) can be solved iteratively, as follows. At halffilling and zero temperature, the average density of electrons with spin $\sigma$ at atom $i$ is defined as $\left\langle n_{i, \sigma}\right\rangle=\sum_{n=1}^{N / 2} \rho_{i, \sigma}\left(E_{n}\right)$, where $N$ is the total number of eigenstates with a given spin, and $\rho_{i, \sigma}\left(E_{n}\right)=\left|\psi_{i, \sigma}^{n}\right|^{2}$ is the local density of states (LDS) at site $i$ for the $n$th eigenstate $\psi_{i, \sigma}^{n}$. Starting from an initial condition of $\left\langle n_{i, \bar{\sigma}}\right\rangle$ for spin $\bar{\sigma}$, the Hamiltonian in (2) is complete for spin $\sigma$ and yields a new set of eigenstates $\left\{E_{n}, \psi_{\sigma}^{n}, n=1, \ldots, N\right\}$, which can then be used to calculate $\rho_{\sigma}\left(E_{n}\right)$, leading to a new set of $\left\langle n_{i, \sigma}\right\rangle$. Using $\left\langle n_{i, \sigma}\right\rangle$ as the initial condition, the Hamiltonian in (2) can be used to solve the set of eigenstates for spin $\bar{\sigma}$, yielding a new set of average density $\left\langle n_{i, \bar{\sigma}}\right\rangle$ of electrons for spin $\bar{\sigma}$. We then iterate the process until $\left\langle n_{i, \sigma}\right\rangle$ and $\left\langle n_{i, \bar{\sigma}}\right\rangle$ reach a steady state. To be concrete, we choose the initial configuration of the system to be that of an antiferromagnetic state, where the initial values of $\left\langle n_{i \downarrow}\right\rangle$ are chosen to be $+1 /-1$ at sublattice $A / B$, respectively. The local spin density at site $i$ is $m_{i}=\left(\left\langle n_{i, \uparrow}\right\rangle-\left\langle n_{i, \downarrow}\right\rangle\right) / 2$. In our model, the number of electrons is fixed, so the total spin density of the whole system is given by $M=\sum_{i=1}^{N} m_{i}=M^{L}+M^{R}=0$, where $M^{L}$ and $M^{R}$ represent the total spin density at the left and the right sides of the potential barrier, respectively.

\section{B. Integrable, chaotic, and nonhyperbolic domains}

We consider four types of geometrical domains with distinct classes of classical dynamics, as illustrated in Fig. 1. For meaningful comparison of results, we set the sizes of the billiards to be approximately the same. The first type is rectangular graphene billiard with integrable classical dynamics. The parameters of the system are $L=161 a=22.86 \mathrm{~nm}$ (armchair boundaries) and $D=85 a=12.05 \mathrm{~nm}$ (zigzag boundaries), where $a=1.42 \AA$ is the distance between two neighboring carbon atoms and the lattice constant is $a_{0}=\sqrt{3} a$. The total number of atoms is $N=10692$. The second type is stadium billiard with parameters $L=22.862 \mathrm{~nm}, D=12.052 \mathrm{~nm}$, and $N=9452$. The underlying classical dynamics is chaotic but with an infinite number of neutrally stable periodic orbits, corresponding to particles bouncing back and forth vertically at the rectangular portion of the billiard. The third type is bowtie billiard, which is cut from a rectangle graphene sheet of $14.5 \mathrm{~nm}$ by $7.2 \mathrm{~nm}$ by circles of radius $r=32.7 \mathrm{~nm}$, and the domain contains 10946 carbon atoms. For the bowtie billiard, the classical dynamics is fully chaotic (hyperbolic) with all periodic orbits being unstable. The fourth type is the mushroom billiard with a mixed classical phase space (nonhyperbolic classical dynamics [65,74]), i.e., there are coexisting chaotic sets and KAM tori. The radius of the semicircle is $R=11.86 \mathrm{~nm}$, and the stem of the mushroom has the sizes $11.86 \mathrm{~nm}$ by $5.93 \mathrm{~nm}$. The total number of carbon atoms contained in the mushroom billiard is 10620 .

In the quantum regime, classical chaos is fundamentally suppressed due to the quantum uncertainty or finite Planck constant that effectively leads to "discretization" of the classical phase space. This should be contrasted with the discretization of the physical or configuration space through a crystal lattice. In general, any such discretization scheme is incompatible with chaos because classically the Planck constant is effectively zero and motion can occur on arbitrarily fine scales, but this difficulty is somewhat alleviated due to the finite Planck constant in the quantum regime. Only in the limit of large geometric dimensions as compared to the lattice constant will the effect of chaos be manifested in the quantum behaviors. For the geometrical domains shown in Fig. 1, we test different sizes and find that the pertinent quantum phenomena are quantitatively the same insofar as the system is sufficiently large, e.g., with more than 7000 atoms.

For all four types of billiards, a thin potential barrier is placed along a symmetric line of the system. Let $x$ specify the direction perpendicular to the symmetric line, the potential function can be written as $V(x)=V_{0}[\Theta(x-L / 2+$ $w / 2)-\Theta(x-L / 2-w / 2)] / 2$, where $\Theta$ is the Heaviside step function. Effectively, the whole billiard system thus has a double quantum-well (QW) structure. We set (somewhat arbitrarily) $V_{0}=0.766 t$ and $w=2.5 a$ in all calculations.

\section{Characterization of tunneling}

For nonrelativistic single particle quantum systems, the tunneling phenomenon and the effect of classically chaotic dynamics can be conveniently studied by considering symmetric quantum billiards with a potential barrier placed along the line of symmetry. In such a situation, the eigenstates are either symmetric or antisymmetric, and they appear in pairs. A particle will tunnel through completely from one side to another, and then back and forth, generating an oscillating pattern. The tunneling dynamics can then be fully characterized by the tunneling rate, which can be expressed in terms of the corresponding energy splittings of the symmetricantisymmetric tunneling pairs [73]. In relativistic quantum systems, the symmetric and antisymmetric eigenstates do not necessarily come in pairs. In this case, the tunneling rate can be calculated, for each eigenstate, by setting a special type of corresponding initial state localized on one side of the barrier. The tunneling rate can be determined through the time evolution of such an initial state. This method is general, as for nonrelativistic quantum tunneling systems, the results agree completely with those calculated by the symmetric-antisymmetric energy splitting method [58]. When many-body interactions are included, the method needs to be 
modified further. Especially, the interactions can effectively be represented by a mean-field potential $U\left\langle n_{i, \bar{\sigma}}\right\rangle$ for a $\sigma$ electron. With respect to tunneling, there is then an extra potential that is different for spin-up and spin-down electrons. This extra potential breaks the mirror symmetry and induces localized states with diminishing tunneling. As a result, an additional quantity, the tunneling probability, together with the tunneling rate, is needed to fully describe the tunneling phenomena in relativistic many-body quantum systems.

To be specific, the method developed for single-electron tunneling in Dirac fermion and graphene systems [58,65] can be readily adopted to systems described by the Hubbard Hamiltonian. First, we solve the eigenenergy values and eigenstates from $H_{\sigma}|n\rangle_{\sigma}=E_{n \sigma}|n\rangle_{\sigma}$, where $H_{\sigma}$ is the meanfield Hamiltonian in Eq. (2) and $\left\langle n_{i, \bar{\sigma}}\right\rangle$ are the steady-state values. An arbitrary state $|\psi\rangle_{\sigma}$ can then be written as a linear combination of the eigenstates $|\psi\rangle_{\sigma}=\sum_{n} a_{n}|n\rangle_{\sigma}$, where $a_{n}$ denotes a set of normalized coefficients. Second, for each eigenenergy value $E_{n \sigma}$ and its associated eigenstate, we define a new wave function $\bar{\psi}_{n, \sigma}(\boldsymbol{r})$, such that the corresponding probability is concentrated entirely on one side of the barrier, say the left side:

$$
\bar{\psi}_{n, \sigma}(x, y)= \begin{cases}C|n\rangle_{\sigma}, & x \leqslant(L-w) / 2 \\ 0, & \text { otherwise }\end{cases}
$$

where $C$ is a normalization constant. This new, asymmetrical wave function can be expanded in the original base of eigenstates: $|\bar{\psi}\rangle_{n, \sigma}=\sum_{k} b_{k}|k\rangle_{\sigma}$, where $b_{k}$ is a set of expansion coefficients given by $b_{k}=\langle k \mid \bar{\psi}\rangle_{\sigma}$. The time evolution of the wave function $|\bar{\psi}\rangle_{n, \sigma}$ is then given by

$$
|\bar{\psi}(t)\rangle_{n, \sigma}=\sum_{k} b_{k} e^{-i E_{k} t / \hbar}|k\rangle_{\sigma} .
$$

In general, $b_{n}$ is significantly larger than other coefficients. Depending on the original state, there can be two, three, or a few coefficients that are well separated from the rest of the coefficients that are negligible. As a result, the summation of Eq. (4) can effectively be evaluated using a small number (usually tens) of states with appreciable coefficients.

Starting from Eq. (4), the left-well probability $P_{\sigma}^{L}(t)$ can be written as

$$
P_{\sigma}^{L}(t)=\langle\bar{\psi}(t) \mid \bar{\psi}(t)\rangle_{n, \sigma}^{L}=\sum_{k, k^{\prime}=1}^{N} b_{k} b_{k^{\prime}}\left\langle k \mid k^{\prime}\right\rangle_{\sigma}^{L} e^{-i\left(E_{k^{\prime}}-E_{k}\right) t},
$$

where the upper index $L$ indicates integration over the left well only and $b_{k}$ is real. Since $|\psi(t)\rangle_{n, \sigma}$ can be approximated by a few terms, the sum in Eq. (5) can be approximated by a few terms as well. From direct numeric calculation, we find that most of the states can be approximated by either two or three eigenstates, with few exceptions.

Say, initially, we choose an initial state entirely in the left well: $P^{L}(t=0)=\langle\bar{\psi}(0) \mid \bar{\psi}(0)\rangle_{\sigma}^{L}=1$, where the upper index $L$ denotes the integration over the region on the left side of the barrier, i.e., $x \leqslant(L+w) / 2$. For $t>0$, the tunneling process begins so that the probability $P^{L}(t)$ decreases with time and reaches its first minimum value $P_{\min }^{L}$ at time $t=\Delta T$. The tunneling rate is conveniently determined by [58] $R=1 / \Delta T$, where the Planck's constant has been normalized to unity: $\hbar=1$. Defining $\Delta P=1-P_{\min }^{L}$, we see that $\Delta P$ is the portion that tunnels to the right side of the barrier. The rate $R$ and tunneling probability $\Delta P$ characterize the tunneling process completely.

Similarly, one can choose an initial state that is localized in the right-hand side of the barrier: $P^{R}(t=0)=$ $\langle\bar{\psi}(0) \mid \bar{\psi}(0)\rangle_{\sigma}^{R}=1$, examine the time evaluation $P^{R}(t)$, and determine the tunneling rate accordingly. Due to symmetry we have $P_{\sigma}^{L}=P_{\bar{\sigma}}^{R}$, so it is necessary to focus only on the tunneling from the left side for spin-up and spin-down states to obtain a complete picture of the quantum tunneling dynamics.

\section{RESULTS}

\section{A. Polarization of spin wave functions associated with confined states}

To gain intuition we first study confined states in the absence of any potential barrier for the rectangular geometry. Representative results are shown in Fig. 2(a), where the profiles of the probability density in the horizontal direction for electrons with different spins are presented. From Fig. 2(a) we observe that the confined states are not polarized, i.e., the spin-up and spin-down wave functions are nearly identical in their spatial distributions in the entire domain. The small difference between the spin-specific wave functions diminishes

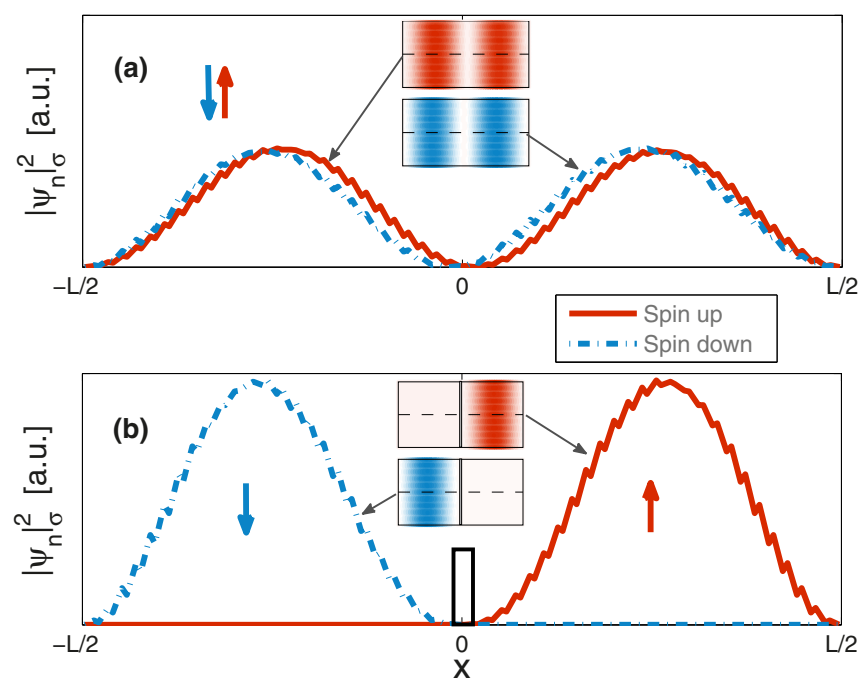

FIG. 2. (Color online) Representative confined eigenstates associated with spin-up (red/solid curves) and spin-down (blue/dashed curves) electrons: (a) Probability density profile of the 5849th eigenstate at $y=D / 2$ without a potential barrier, where it extends in both potential wells. (b) Probability density profile of the 5860th eigenstate at $y=D / 2$ with a potential barrier of height $V_{0}=0.766 t$ and width $w=2.5 a$ at $x=0$ (represented by the gray rectangle). In (b) there is spin polarization, i.e., spin-up electrons reside in the right well and spin-down electrons reside in the left well. The corresponding eigenenergies are $E_{5849} \approx 0.6819 t$ for (a) and $E_{5860} \approx$ $0.6924 t$ for (b). The insets in both panels show the corresponding LDS patterns in the entire domain. Note that the wave functions have a zigzag appearance because they are plotted for both graphene sublattices, denoted by $A$ and $B$. (A plot of the wave function on one sublattice would appear more smooth.) 
for $E \rightarrow t$, where $t$ is the nearest-neighbor hopping energy in the graphene lattice.

We next investigate the case where there is a narrow potential barrier at the center of the rectangular graphene flake. A surprising phenomenon is that, due to a combined effect of electron-electron interactions and the potential barrier, there are eigenstates in which the spin-up and spin-down electrons become strongly polarized. For example, for the case shown in Fig. 2(b), the spin-down (up) electrons tend to focus on the left (right) side of the barrier only. While the case shown in Fig. 2(b) corresponds to wave vector $k_{x} \approx 2 \pi / L$, other polarized states can be found for $k_{x} \approx \pi n / L(n=4,6, \ldots)$. This polarization phenomenon occurs only for the original eigenstates of even parity $(n=2,4,6, \cdots)$ in the absence of potential barrier. For eigenstates of odd parity $(n=1,3,5, \ldots)$ originally, introduction of a potential barrier does not generate spin polarization.

The polarized states appear in pairs. For example, if there is a polarized state in which the spin-up electrons concentrate on the left quantum well (spin-down electrons on the right well), there will be a corresponding polarized state that somewhat mimics a reflected version of the original state, e.g., represented by a wave function for which the spin-up electrons concentrate on the right well. The energy difference between the paired states are typically small. Figure 3(a) shows the energy levels of the polarized-state pairs. The red and blue bars with an arrow (indicating spins) located at the left or the right side denote that the electrons are mainly polarized at the left or the right quantum well, respectively. For the specific initial condition used, the average local spin density is positive at the right boundary, and negative at the left boundary. Because of the repulsive Coulomb interaction between the electrons, the polarized state with spin-up electrons residing at the right well has a lower energy, while its counterpart has a higher energy. Typical polarized states are shown in Figs. 3(b) and 3(c).
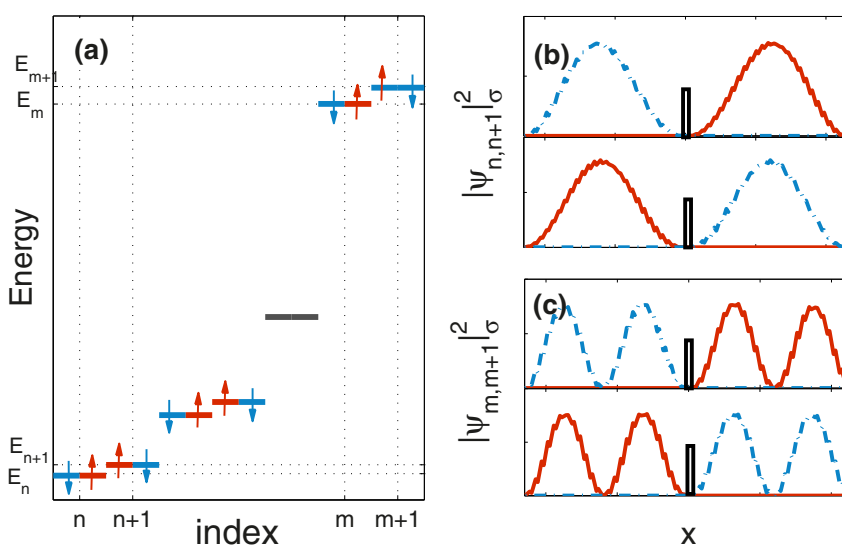

FIG. 3. (Color online) (a) Eigenenergy levels versus the eigenstate index. The red and blue bars with up and down arrows, respectively, represent a pair of polarized states $(n=5760$ and $m=$ 5765), where the corresponding eigenenergies are $E_{n}=0.6203 t$, $E_{n+1}=0.6204 t, E_{m}=0.6235 t$, and $E_{m+1}=0.6237 t$. The black bar without any arrow corresponds to a nonpolarized state. (b) and (c) Probability densities for the polarized state pairs $(n, n+1)$ and $(m$, $m+1)$, respectively. Note that the energy difference for each pair is quite small.
The accumulation of edge states contributes to the polarized average spin density (magnetic moments) of the graphene sheet. When polarization of the confined states emerges, the polarized magnetic moments remain approximately the same. The reason is that, for each pair, the magnetic moments of the polarized states nearly cancel each other.

The basic physical mechanism for the emergence of the localized spin-polarized states can be understood by employing the Dirac equation [44-50]:

$$
-i v_{F}[\boldsymbol{\sigma} \cdot \boldsymbol{p}+\tilde{V}] \boldsymbol{\psi}=E \boldsymbol{\psi},
$$

where $v_{F}$ is the Fermi velocity of electrons in graphene, $\sigma=$ $\left(\sigma_{x}, \sigma_{y}, \sigma_{z}\right)$ are the Pauli matrices, the components of the spinor wave function $\boldsymbol{\psi}=\left[\psi_{A}, \psi_{B}\right]$ correspond to the pseudospins that characterize whether the electron resides on sublattice $A$ or $B$, respectively, and the term $\tilde{V}$ represents the mean-field potential due to a combined effect of the barrier potential and the edge potential caused by the intrinsic magnetic moments of spin electrons. It can be demonstrated that the Coulomb repulsive interaction leads to antiferromagnetic steady states (mostly edge states) at the graphene sublattices, with net spin-up electrons residing on the zigzag boundary on one side, while spin-down electrons residing on the other zigzag boundary. These steady states can in turn be regarded as an effective potential that breaks the left-right reflection symmetry. However, this symmetry breaking can be subdued as a shift of the system. When a potential barrier is applied, it introduces into the system a natural reference point that makes this shift unlikely, yielding spin-polarized confined states. These states often appear in pairs so that their magnetic moments cancel each other. Details of the solutions of the Dirac equation and their physical analysis are presented in Sec. IV.

\section{B. Tunneling rate and probability of spin-polarized states}

When a potential barrier is placed in a closed domain, electrons can tunnel through from one side of the barrier to another. For graphene systems, a systematic method for numerically calculating the tunneling rate has been developed recently [58] in the single-electron, tight-binding framework.

Our numerical computation reveals a striking phenomenon: when electron-electron interactions are present, the tunneling probability $\Delta P$, the portion of the state that can tunnel into the other side of the barrier, can be extremely small, e.g., $\Delta P \sim 10^{-4}$, as shown in Fig. 4(a). This should be contrasted to the case of single-electron tunneling, where the value of $\Delta P$ is on the order of unity, as shown in Fig. 4(b). Corresponding to the extremely small values of $\Delta P$, the tunneling rate $1 / \Delta T$ that characterizes the "speed" of tunneling assumes also extremely small values. The reason that $\Delta P$ can be so small for electrons with Coulomb interactions lies in the emergence of the spin-polarized states. In particular, in a single-electron tunneling system, because of the left-right reflection symmetry, the eigenfunction also has even or odd symmetry, and the probabilities for the electron to reside in the left and right wells are equal. When renormalizing the left part of the wave function for the situation where the electron initially is located in the left well, the symmetry stipulates that the electron will eventually tunnel to the right side as 

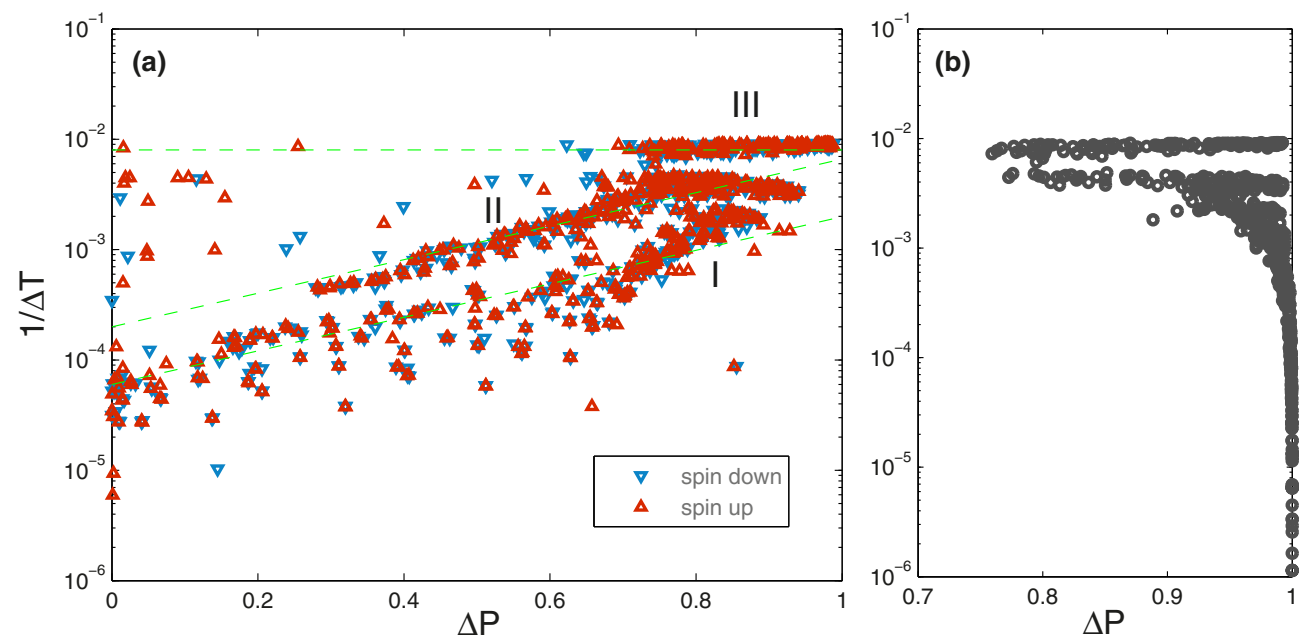

FIG. 4. (Color online) Tunneling rate $1 / \Delta T$ versus $\Delta P$ for the rectangular graphene billiard for two cases where (a) there are electronelectron interactions as described by the Hubbard model and (b) there is only a single electron in the system.

there is no mechanism to contain it only within the left well. However, for the many-body case where electron-electron interactions are present, the graphene zigzag edges at the opposite boundaries bear different magnetic moments, positive on one side and negative on the other side. Spin-up and spin-down electrons will then "feel" different potentials at the boundaries, leading to the left-right reflection symmetry breaking and, consequently, to the emergence of spin-polarized states. For example, consider an eigenstate associated with spin-up electrons residing mostly in the left well. Because it is an eigenstate, the time evolution of the probability in the left well $P^{L}$ will be a constant and approximately equal to 1. That is, it is a spin-polarized and localized state. We can then deduce that, for spin-up electrons in the left well initially, the associated state will have a significant component in the spin-polarized states, with a high probability $P^{L}$ in the left well at all times resulting in extremely small values of $\Delta P$. For comparison, we have also computed the tunneling rate for the single-electron case, as shown in Fig. 4(b). In this case, while the tunneling rate can be small, the tunneling probability $\Delta P$ is generally large. We note that, in addition to the strongly localized states, there are also states that are less localized, resulting in relatively larger values of $\Delta P$. These states can be either spin polarized or nonpolarized.

A careful examination of Fig. 4(a) reveals that the data points can be categorized into three classes, depending on the patterns of their distribution in the $(1 / \Delta T, \Delta P)$ plane. These different tunneling behaviors correspond to distinct time evolutions of the probability $P^{L}(t)$ for electrons in the left well. In particular, for classes I and II in Fig. 4(a), $P_{L}(t)$ is a cosine function, while for class III, $P_{L}(t)$ is a zigzag curve. In Sec. IV we develop a theory to understand the distinct behaviors in the time evolution of $P_{L}$.

\section{Regularization of tunneling by chaos}

Our understanding of the spin-polarized confined states in the rectangular graphene indicates that many-body interactions are key to the emergence of such states. These states have a significant effect on the quantum tunneling dynamics.
Especially, for the rectangular graphene billiard, not only can the tunneling rate be negligibly small (corresponding to large values of $\Delta T$ ), but also the tunneling magnitude $\Delta P$. While our analysis suggests that other physical factors, such as the potential barrier and the pseudospin degree of freedom of graphene, also play a role in the formation of the localized spin-polarized states, many-body interactions are the single most important factor. This is because, without such interactions, the tunneling rate and magnitude can typically be appreciable in graphene systems of the same geometry [58]. Does this mean that, in any realistic graphene system where many-body interactions are inevitably present, tunneling is far less likely? If this were the case, it would be difficult to develop a graphene-based tunneling device. Here we shall show that this difficulty can be overcome by taking advantage of classical chaos. In particular, we demonstrate that, when the geometry of the domain is such that the corresponding classical dynamics is chaotic, both the tunneling rate and magnitude can be significantly enhanced, suggesting that chaotic geometries are favorable if fast and sizable tunneling is needed when developing graphene tunneling devices. Since the effective geometrical shape of the domain can be modulated by external means, such as atomic probe [84] or gate voltage, control of quantum tunneling dynamics in the presence of many-body interactions can be achieved by using chaos.

Figure 5 shows the tunneling rates for a stadium-shaped and a bowtie-shaped graphene billiards, where the latter is fully chaotic in that all classical periodic orbits are unstable. As compared with the rectangular billiard, for both types of chaotic billiards, the points in the plot of the tunneling rates $1 / \Delta T$ versus $\Delta P$ in Fig. 5 are more concentrated in both dimensions. The concentration is more compact for the bowtie billiard (the "more chaotic" domain). More specifically, some pronounced features of Fig. 5 are the following.

First, as compared with the rectangular domain, the tunneling rates associated with the chaotic domains are greatly enhanced, e.g., from about $10^{-6}$ in Fig. 4 to about $10^{-4}$ in Fig. 5. Comparing Fig. 5(a) with 5(b), we see that overall, the tunneling rates for the more chaotic bowtie billiard are even larger. 

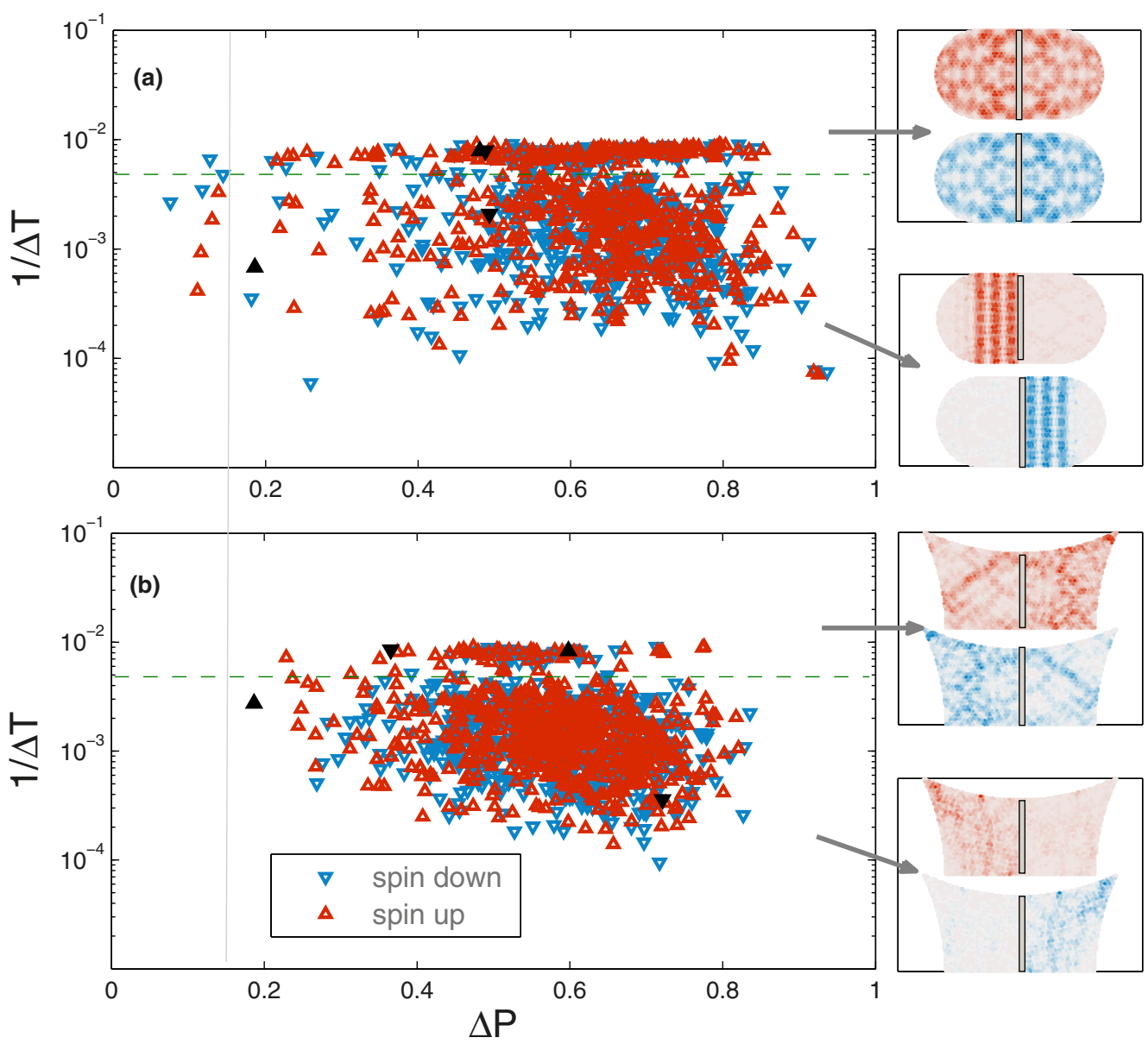

FIG. 5. (Color online) Tunneling rates versus $\Delta P$ for chaotic geometries: (a) Stadium and (b) bowtie graphene billiards. The green dashed horizontal line indicates the separation of the two classes of spin-polarized (lower) and unpolarized (upper) states. The black solid up- and down-triangles correspond to the typical LDS patterns in each class for spin-up and spin-down states, respectively.

Second, the range of $\Delta P$ is reduced in the chaotic cases in the sense that, as compared with the rectangular domain, the minimum value of $\Delta P$ is larger and its maximum value becomes smaller. For the stadium and bowtie domains, the ranges are from 0.1 to 0.9 and from 0.2 to 0.8 , respectively. A common feature is that the localized states with extremely small values of tunneling rate no longer exist. In fact, the maximal local spin densities are comparable for all three types of domains: $0.1565,0.1507$, and 0.1691 for rectangular, stadium, and bowtie billiards, respectively. Thus, the nearly perfect spin-polarized states in the integrable domain have been effectively eliminated by chaos!

Third, for the integrable case, as shown in Fig. 4, the points in the plot of $1 / \Delta T$ versus $\Delta P$ are grouped into three classes. For both types of chaotic domain, as shown in Fig. 5, the points only belong to two classes. The first class is for $1 / \Delta T \sim 10^{-2}$, which is similar to case III for the rectangular domain in that the eigenstates are not polarized but distributed equally between the left and the right wells. It might be tempting to regard the lower points in Fig. 5 (especially in the side panels) as corresponding to some sort of spin-polarized states. However, in contrast to the rectangular case in which the states are either antiphase (class I) or having identical phases for $A$ and $B$ atoms (class II), the eigenstates for the chaotic cases do not have such a clear cut for the relative phases, but assume randomized values between 0 and $\pi$.

We conclude that, when electron-electron interactions are taken into account in a graphene system, chaos cannot only regularize the tunneling rate as in situations where such interactions are neglected [58,65,73], but also regularize the polarization and mix the relative phase between the states associated with the two distinct sublattices.

In realistic quantum devices, nonhyperbolic dynamics with mixed phase space [33-43] can be expected to arise typically. Here we investigate the tunneling dynamics in classically nonhyperbolic systems in the presence of electronelectron interactions. We choose the mushroom billiard, a mathematically proved nonhyperbolic system [74], as shown in Fig. 1(d). A potential barrier is placed along the vertical symmetric line. Figure 6 shows the tunneling rate $1 / \Delta T$ versus $\Delta P$. The points are scattered in three regions, which are separated by the horizontal dashed lines. The middle and the lower parts are similar to the two regions for the chaotic cases in Fig. 5. Specifically, the middle part corresponds to nonpolarized states, while the lower part corresponds to the polarized states. The upper region, which is absent for both integrable and chaotic cases, corresponds to the integrable part of the mushroom billiard in the stem region. From Fig. 6 


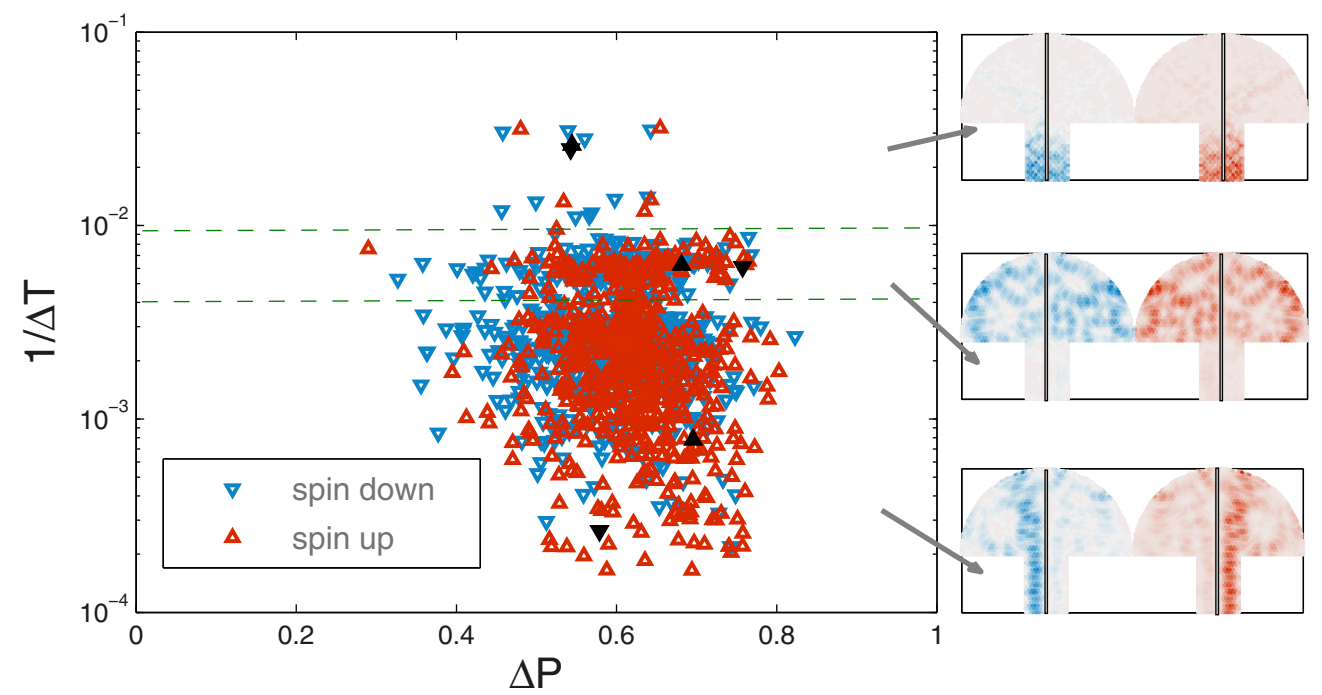

FIG. 6. (Color online) Tunneling rates versus $\Delta P$ for the nonhyperbolic mushroom billiard. The green dashed horizontal lines indicate the separation of the data points into three different classes. The middle and lower classes correspond to the chaotic components. The upper class is originated from the stem of the mushroom billiard. The black solid up- and down-triangles correspond to the LDS patterns for spin-up and spin-down states in the right-side panels, respectively.

the minimum value for $\Delta P$ is about 0.3 , which is larger than those for both chaotic cases. For the mushroom billiard, the classical periodic orbits generally cross both the left and the right parts, thus strong polarized states are less likely to form, leading to relatively larger values of $\Delta P$. These results are quantitatively similar to those in absence of electron-electron interactions [65].

\section{THEORY}

\section{A. Emergence of polarized states: Mean-field theory}

The unit cell of the honeycomb lattice of graphene has two nonequivalent carbon atoms, $A$ and $B$, resulting in two Dirac points in the wave vector space: $\boldsymbol{K}\left(\boldsymbol{K}^{\prime}\right)=\left(2 \pi / \sqrt{3} a_{0}, \pm\right.$ $\left.2 \pi / 3 a_{0}\right)$. In the vicinity of a Dirac point, the dispersion relation is linear: $E= \pm \hbar v_{F}|\boldsymbol{k}|$, where $v_{F}$ is the Fermi velocity of electrons in graphene, and $\boldsymbol{k}$ denotes the deviation in the momentum from the Dirac point. Neglecting the coupling between the two Dirac points, the quantum dynamics of the quasiparticles in graphene are described by the Dirac equation [44-50], Eq. (6). The electron-electron interaction can be treated by the mean-field Hubbard Hamiltonian. In particular, the states of electrons of spin $\sigma$ are affected by the mean-field electron density of the opposite spin $\left\langle n_{i, \bar{\sigma}}\right\rangle$. This density has appreciable values only at the zigzag edges and it is in fact exponentially small inside the domain [Fig. 7(a)]. As a result, effectively the electron-electron interaction can be described by the following square potential function $\varepsilon_{\sigma} M(x)$ [Fig. 7(b)]:

$$
M(x)= \begin{cases}+M_{0}, & x<\Delta l \\ -M_{0}, & x>L-\Delta l \\ 0, & \text { otherwise }\end{cases}
$$

where $\varepsilon_{\sigma}=\varepsilon_{\uparrow(\downarrow)}=+1(-1)$ for spin up (down), $M_{0}$ is the effective value of $\left(U\left\langle n_{\sigma(\bar{\sigma})}\right\rangle\right)$ close to the zigzag boundary, $U$ is a parameter characterizing the Coulomb interaction in the mean-field Hubbard Hamiltonian, and $\Delta l \sim a$ is the width of the effective potential. Note that the effective potential felt by the spin-up and spin-down electrons are opposite to each other. The overall potential for spin $\sigma$ electrons can thus be

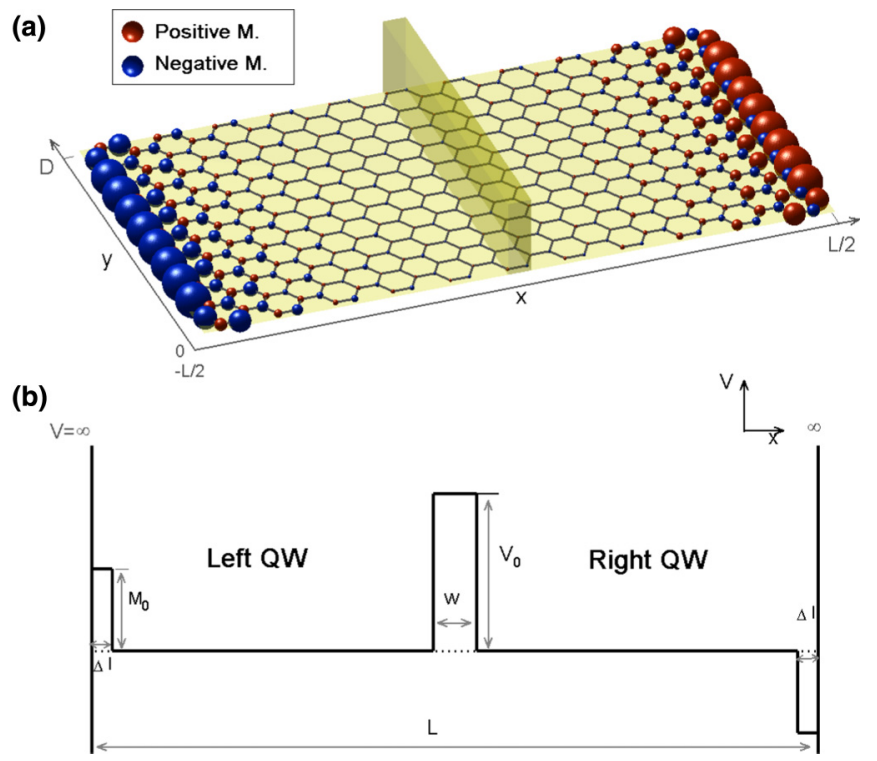

FIG. 7. (Color online) (a) Illustration of a rectangular graphene domain of length $L$ and width $D$ with a potential barrier in the middle. The zigzag and armchair edges are along the $x$ and $y$ axes, respectively. The filled red (blue) circles at the right (left) zigzag boundaries denote the positive (negative) magnetic moments, the radii of which represent the strength of the spin density. (b) The effective potential profile $\widetilde{V}_{\uparrow}(x)$ at the position $y=D / 2$ for spin-up electrons, where the positive and negative potentials near the boundaries represent the strength of the respective spin density, which approach the value of $M_{0}\left(-M_{0}\right)$ at the left (right) zigzag edges in (a). The effective potential for spin-down electrons is reversed at the boundaries as compared with that for spin-up electrons. 
expressed as $\widetilde{V}_{\sigma}=V(x)+\varepsilon_{\sigma} M(x)$, and the mean-field Dirac Hamiltonian near the Dirac point $\boldsymbol{K}\left(\boldsymbol{K}^{\prime}\right)$ is given by

$$
H_{\boldsymbol{K}\left(\boldsymbol{K}^{\prime}\right), \sigma}=v_{F}\left(\begin{array}{cc}
\widetilde{V}_{\sigma} & -i \partial_{x} \mp \partial_{y} \\
-i \partial_{x} \pm \partial_{y} & \widetilde{V}_{\sigma}
\end{array}\right) .
$$

For notational clarity, we shall use small letters $\epsilon, \widetilde{v}$, and $m$ to denote the energy $E$, potential $\widetilde{V}$, and $M$ divided by $\hbar v_{F}$. Note that the Hamiltonian with the effective potential $\varepsilon_{\sigma} M(x)$ no longer has the left-right reflection symmetry. However, it has the combined symmetry of simultaneous reflection and spin interchange. It is thus only necessary to consider spin-up electrons, because the states of spin-down electrons can be obtained straightforwardly by the symmetry operation $\psi_{\downarrow}(x)=\psi_{\uparrow}(-x)$.

For the $\boldsymbol{K}\left(\boldsymbol{K}^{\prime}\right)$ valley, the bulk eigenstate of the Dirac Hamiltonian Eq. (8) is given by [85]

$$
\left[\psi_{A}^{(\prime)}, \psi_{B}^{(\prime)}\right]^{T}=e^{i \boldsymbol{k} \cdot \boldsymbol{r}}\left[1, \mp e^{ \pm i \phi}\right]^{T}
$$

associated with energy $E= \pm \hbar v_{F}|\boldsymbol{k}|$, or $\epsilon= \pm|\boldsymbol{k}|$, where $\phi=$ $\tan ^{-1} k_{y} / k_{x}$. Under the influence of the total potential $\widetilde{V}$, the wave vector in the $x$ direction becomes

$$
k_{x}=\sqrt{(\epsilon-\widetilde{v})^{2}-k_{y}^{2}} .
$$

The solutions of the Dirac equations for the armchair or zigzag graphene nanoribbons can be found in Refs. [48,86]. For the armchair boundaries, the wave vector $k_{y}$ is related to the length $D$ in the $y$ direction by $k_{y} \longrightarrow k_{n}=n_{y} \pi / D-4 \pi / 3 a_{0}$, where $n_{y}=0, \pm 1, \pm 2, \ldots$. For an armchair nanoribbon, $k_{n}$ is thus independent of the potential $\widetilde{V}$ and $k_{x}$, and the wave function in the $y$ direction can be separated as $\left[\psi_{A}, \psi_{B}\right]^{T}=$ $e^{i k_{n} y}\left[\phi_{A}, \phi_{B}\right]^{T}$, where

$$
\left(\begin{array}{c}
\phi_{A} \\
\phi_{B}
\end{array}\right)=\left(\begin{array}{c}
\frac{1}{\epsilon_{\gamma}}\left[a_{\gamma}\left(k_{n}-z\right) e^{z x}+b_{\gamma}\left(k_{n}+z\right) e^{-z x}\right] \\
a_{\gamma} e^{z x}+b_{\gamma} e^{-z x}
\end{array}\right) .
$$

The coefficients $a_{\gamma}$ and $b_{\gamma}$ in Eq. (9) represent the amplitudes of the wave function in the region $\gamma$ and $\epsilon_{\gamma}=\epsilon-\widetilde{v}$ is the relative energy, where $\gamma=L, R, C$ stand for the left, the right, and the central barrier regions, respectively. For confined and edge states, we have $z=k_{x}$ and $z=i k_{x}$, respectively.

For zigzag graphene ribbons, the wave vector $k_{x}$ parallel to the armchair edges couples with $k_{n}$ if the potential $\tilde{v}$ is equal to zero or a constant. In this case, the relationship between the two wave vectors in orthogonal directions is given by [86]

$$
\left(k_{n}-z\right) /\left(k_{n}+z\right)=\exp (\mp 2 L z),
$$

for the $\boldsymbol{K}\left(\boldsymbol{K}^{\prime}\right)$ point, respectively. A simplified condition of $k_{x}$ for confined states can be rewritten as [48,86] $k_{x}=$ $\pm \tan ^{-1}\left(k_{x} / k_{n}\right)$ for the $\boldsymbol{K}\left(\boldsymbol{K}^{\prime}\right)$ valley.

We first focus on the solution in the $\boldsymbol{K}$ valley and the case without central barrier. In this case, $k_{n}$ is determined by the width $D$ of the domain but, due to the effective potential $M(x)$ close to the zigzag boundaries, it is infeasible to obtain an exact expression for $k_{x}$. Note that the width of this potential $\Delta l$ is typically much smaller than the length $L$ of the device and is also smaller than the wavelength in the $x$ direction. The following approximations can then be justified. In particular, we assume that the ratio between the wave amplitude is a constant: $a_{\gamma} / b_{\gamma}=-1$, and the potential $\tilde{v}$ is also a constant for the whole system, which are valid for the case without the central potential barrier. Within distance $\Delta l$ to the boundaries $x=-L / 2$ and $L / 2$, the wave function can then be written as

$$
\left(\begin{array}{c}
\phi_{A} \\
\phi_{B}
\end{array}\right) \approx a_{\gamma}\left(\begin{array}{c}
\frac{1}{\epsilon_{\gamma}}\left[-2 z+2 k_{n} z x\right] \\
z x
\end{array}\right)
$$

by first-order linear approximation. In these regions, the only difference from the case where the effective potential is absent lies in the wave vector or the wavelength, i.e., $k_{x}^{ \pm}=\sqrt{(\epsilon \pm m)-k_{n}^{2}}$. Since what matters here is only the phase change in the wave function, we can assume that the wave vector is unchanged but the length of this potential region is changed, say from $\Delta l$ to $\Delta \widetilde{l}^{ \pm}$for the right and left boundaries, respectively. We have

$$
\Delta \widetilde{l}^{ \pm}=\left(k_{x} / k_{x}^{ \pm}\right) \Delta l
$$

As a result, the wave vectors $k_{\gamma}$ takes on the same value as for the case without the potential $m$ and the only difference is the change in the effective width $\Delta l$. In particular, at the left boundary, $\Delta l$ shrinks to $\Delta \widetilde{l}^{-}$and, at the right boundary, $\Delta l$ expands to $\Delta \widetilde{l}^{+}$. This is illustrated in Fig. 8(a) in the zoom-in insets, which show the comparison between the real wave function (black solid curve) and the effective wave function (red dashed curve) in the potential regions. Based on these
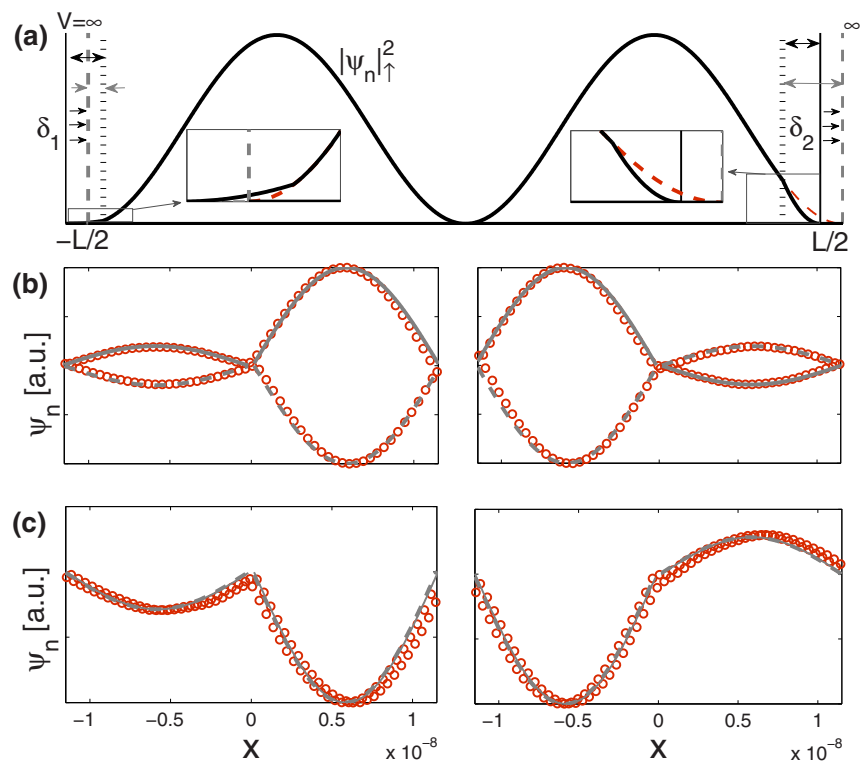

FIG. 8. (Color online) (a) Illustration of the shift in the wave function caused by the effective potential $M(x)$. The black solid vertical lines are the actual domain boundaries. The gray dashed lines represent the effective boundaries. The black solid curves show the actual probability of an evenly confined spin-up state without the potential barrier, and the red dashed curves represent the effective wave function after the "shift." (b) and (c) Antiphase and in-phase wave functions for sublattice $A$ and $B$, respectively, where red circles represent the simulation results from the mean-field Hubbard Hamiltonian and the gray solid and dashed curves are predictions from theory [Eqs. (9), (11), and (13)]. The boundary shift is $\delta=0.032 a$. The wave function mode in the $y$ direction is chosen to be $n_{y}=16$ for (b) and $n_{y}=112$ for (c). The numerical results are from the (arbitrarily chosen) states $n=5547$ and 5548 for (b) and $n=5568$ and 5569 for (c). 
observations, we can effectively transform the system into a new system without potential $m$, but with the boundaries shifted by a displacement $\delta_{1(2)}$, as shown schematically in Fig. 8(a). The effective boundary displacement can be determined as

$$
\delta_{1}=\Delta l-\Delta \tilde{l}^{-} \text {and } \delta_{2}=\Delta \tilde{l}^{+}-\Delta l .
$$

The physical meaning is that the wave functions of spin-up electrons are shifted toward the right (positive axis) by the amount $\delta=\left(\delta_{1}+\delta_{2}\right) / 2$. We shall see that, because of this relative shift, when a potential barrier is placed in the middle of the domain, the equivalent system with shift breaks the leftright reflection symmetry, which is the key to the emergence of spin-polarized states. Note that, if the energy $\epsilon$ is close to the potential value $m$, the wave vector $k_{x}^{-}$will be purely imaginary and the wave function near the left boundary has an exponential form, which differs from a normally propagating wave function. But this does not affect our analysis.

Next, we consider the case where a potential barrier is placed along the symmetric line of the domain and provide an explanation for the emergence of the spin-polarized states. To be concrete, we choose a narrow potential barrier of width $w=2.5 a=0.016 L$ and place it at $x=0$. From the above analysis, $x=0$ is no longer the center of the equivalent

system, as the boundaries have been shifted to the right by the displacement $\delta$ so that the widths of the left- and right-side quantum wells become $W_{1} \approx(L-w) / 2-\delta$ and $W_{2} \approx(L-w) / 2+\delta$, respectively. Equivalently, the electronelectron interaction system with a symmetric double-well structure can be transformed into a single-electron system with asymmetric double wells.

For an asymmetric double-well system, the standard wave functions for graphene [Eq. (9)] satisfy the boundary conditions: $\phi_{A(B)}^{L}\left(-L_{1} / 2\right)=\phi_{A(B)}^{R}\left(L_{2} / 2\right)=0$, where $L_{1,2}=$ $2 W_{1,2}+w$. We obtain

$$
b^{L}=-a^{L} e^{-i k_{x} L_{1}} \text { and } b^{R}=-a^{R} \frac{k_{n}^{-}}{k_{n}^{+}} e^{i k_{x} L_{2}},
$$

where $k_{n}^{ \pm}=k_{n} \pm i k_{x}, k_{n}=n_{y} \pi / D-4 \pi / 3 a_{0}$, and $k_{x}$ is to be determined. The continuity conditions at the barrier interfaces are

$$
\begin{gathered}
\phi_{A(B)}^{L}(-w / 2)=\phi_{A(B)}^{C}(-w / 2), \\
\phi_{A(B)}^{R}(w / 2)=\phi_{A(B)}^{C}(w / 2) .
\end{gathered}
$$

When Eq. (11) is applied, the equations of $\mathbf{a}=$ $\left[a^{L}, a^{R}, a^{C}, b^{C}\right]^{T}$ can be reorganized as $\mathbf{A} \cdot \mathbf{a}=0$, with $\mathbf{A}$ given by

$$
\mathbf{A}=\left(\begin{array}{cccc}
\frac{\epsilon^{\prime}}{\epsilon}\left[k_{n}^{-} e^{-i k_{x} w / 2}-k_{n}^{+} e^{-i k_{x}\left(-w / 2+L_{1}\right)}\right] & 0 & -k_{n}^{-} e^{-i \beta w / 2} & -k_{n}^{+} e^{i \beta w / 2} \\
e^{-i k_{x} w / 2}-e^{-i k_{x}\left(-w / 2+L_{1}\right)} & 0 & -e^{-i \beta w / 2} & -e^{i \beta w / 2} \\
0 & \frac{\epsilon^{\prime}}{\epsilon}\left[k_{n}^{-} e^{i k_{x} w / 2}-k_{n}^{-} e^{-i k_{x}\left(w / 2-L_{2}\right)}\right] & -k_{n}^{-} e^{i \beta w / 2} & -k_{n}^{+} e^{-i \beta w / 2} \\
0 & e^{i k_{x} w / 2}-\frac{k_{n}^{-}}{k_{n}^{+}} e^{-i k_{x}\left(w / 2-L_{2}\right)} & -e^{i \beta w / 2} & -e^{-i \beta w / 2}
\end{array}\right),
$$

where $\beta=\sqrt{k_{n}^{2}-\epsilon^{\prime 2}}, \epsilon^{\prime}=v_{0}-\epsilon$, and $v_{0}=V_{0} / \hbar v_{F}$ is the barrier height. In order to have nontrivial solutions of a, the matrix A should satisfy the condition $\operatorname{det}|\mathbf{A}|=0$, which in turn solves the only unknown parameter $k_{x}$. In general, a pair of solutions can be found near $2 n \pi / L$, where $n$ is an integer corresponding to the mode number in each quantum well. The final step in our analysis is to obtain the coefficients a. Solving for $a^{L} / a^{R}$, we obtain their relative values as

$$
\begin{aligned}
& a^{L}=\frac{\epsilon^{\prime}}{\epsilon} k_{n}^{-}\left[1-e^{-i k_{x}\left(w-L_{2}\right)}\right]-\left(k_{n}+\beta\right)\left[1-\frac{k_{n}^{-}}{k_{n}^{+}} e^{-i k_{x}\left(w-L_{2}\right)}\right], \\
& a^{R}=e^{\left(i k_{x}+\beta\right) w}\left\{\frac{\epsilon^{\prime}}{\epsilon} k_{n}^{-}\left[1-\frac{k_{n}^{+}}{k_{n}^{-}} e^{i k_{x}\left(w-L_{1}\right)}\right]-\left(k_{n}+\beta\right)\left[1-e^{i k_{x}\left(w-L_{1}\right)}\right]\right\} .
\end{aligned}
$$

The relative values of $b^{L, R}$ can be obtained from Eq. (11). With these coefficients, the wave function in the $x$ direction can be obtained from Eq. (9).

These theoretical predictions can be compared with numerical results from the mean-field Hubbard Hamiltonian for spin-up wave functions, as shown in Figs. 8(b) and 8(c). We obtain a good agreement.

There are several issues associated with experimental realizations. One is the size of the potential barrier. In our simulation, we set the width of barrier to be $0.4 \mathrm{~nm}$, which may be too small for a gate potential to be applied, as the current experimental techniques would allow the minimum width of top gate to be about $10 \mathrm{~nm}$ [87]. Nonetheless, based on the theoretical prediction in Eq. (13), the spin-polarized state is not affected by the width of the central potential barrier: it only depends on the ratio of barrier width $w$ and device length $L$. This allows an experimental study of the localization phenomenon if we enlarge $w$ and $L$ proportionally so that $w$ is sufficiently large, say $15 \mathrm{~nm}$. Another consideration is that the barrier in an experimental realization may not be a square potential but with small deformations. This, however, does not present any serious challenge because the spin-polarized states are stable due to the edge magnetic moments. As a result, the polarized states can still exist and similar regularization effects of chaos should persist.

\section{B. Tunneling rate of spin-polarized states}

From Eq. (4) we have the time evolution of the wave function $|\bar{\psi}\rangle_{n, \sigma}$. Then, the left-well probability $P_{\sigma}^{L}(t)$ is given by Eq. (5). Since $|\psi(t)\rangle_{n, \sigma}$ can be approximated by a few terms, the sum in Eq. (5) can be approximated by a few terms as well. 

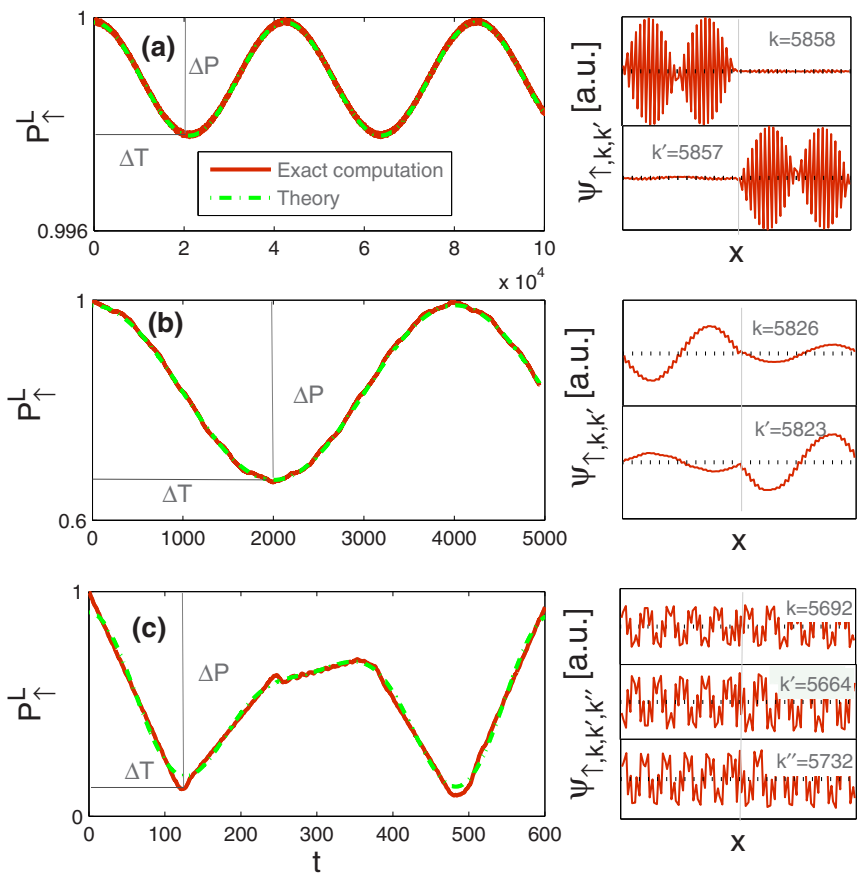

FIG. 9. (Color online) Time evolutions of $P^{L}(t)$ for spin-up states corresponding to $n=5858$ (a), 5826 (b), and 5692 (c), and their projected states with the corresponding weighting coefficients. The green dashed-dotted curves are fitted from Eq. (15) (a) and (b) and Eq. (16) (c).

From direct numerical calculation, we find that most of the states can be approximated by either two or three eigenstates, with few exceptions. Particularly, for classes I and II (e.g., Fig. 4), we can approximate $|\bar{\psi}\rangle_{k, \sigma}$ by two eigenstates only:

$$
|\bar{\psi}\rangle_{k, \sigma} \approx b_{k}|k\rangle_{\sigma}+b_{k^{\prime}}\left|k^{\prime}\right\rangle_{\sigma}
$$

and the expansion coefficients satisfy the relation

$$
b_{k}^{2}+b_{k^{\prime}}^{2} \approx 1
$$

In this case, $|k\rangle_{\sigma}$ and $\left|k^{\prime}\right\rangle_{\sigma}$ are symmetric pairs: $|k(-x)\rangle_{\sigma} \approx$ $\left|k^{\prime}(x)\right\rangle_{\sigma}$, as demonstrated in Figs. 9(a) and 9(b). We then have

$$
\langle\bar{\psi} \mid \bar{\psi}\rangle_{k, \sigma}=\langle\bar{\psi} \mid \bar{\psi}\rangle_{k, \sigma}^{L}=1 \approx b_{k}\langle\bar{\psi} \mid k\rangle_{\sigma}^{L}+b_{k^{\prime}}\left\langle\bar{\psi} \mid k^{\prime}\right\rangle_{\sigma}^{L} .
$$

Comparing this equation with Eq. (14), we get $\langle\bar{\psi} \mid k\rangle_{\sigma}^{L} \approx b_{k}$, and $\left\langle\bar{\psi} \mid k^{\prime}\right\rangle_{\sigma}^{L} \approx b_{k^{\prime}}$. Note that $\langle\bar{\psi} \mid k\rangle_{\sigma}^{L} \approx b_{k}$ is equivalent to $|k\rangle_{\sigma}^{L}=b_{k}|\bar{\psi}\rangle_{\sigma}^{L}$, as the former can be obtained by multiplying $\left\langle\left.\bar{\psi}\right|_{\sigma} ^{L} \text { to the latter. Similarly, we have } \mid k^{\prime}\right\rangle_{\sigma}^{L}=b_{k^{\prime}}|\bar{\psi}\rangle_{\sigma}^{L}$. Substituting these relations back into Eq. (5), we obtain the time evolution of left-side probability as

$$
\begin{aligned}
P_{\sigma}^{L}(t) & \approx b_{k}^{2}\langle k \mid k\rangle_{\sigma}^{L}+b_{k^{\prime}}^{2}\left\langle k^{\prime} \mid k^{\prime}\right\rangle_{\sigma}^{L}+2 b_{k} b_{k^{\prime}}\left\langle k \mid k^{\prime}\right\rangle_{\sigma}^{L} \cos (\Delta E \cdot t) \\
& =b_{k}^{4}+b_{k^{\prime}}^{4}+2 b_{k}^{2} b_{k^{\prime}}^{2} \cos (\Delta E \cdot t),
\end{aligned}
$$

where $\Delta E=E_{k^{\prime}}-E_{k}$. Note that $\left\langle k^{\prime} \mid k^{\prime}\right\rangle_{\sigma}^{L}$ is not zero because the integration is with respect to the left well only. Thus, the $P_{\sigma}^{L}(t)$ curve is a standard cosine-type function. At $t=0$ we have $P_{\sigma}^{L}=b_{k}^{4}+b_{k^{\prime}}^{4}+2 b_{k}^{2} b_{k^{\prime}}^{2}=\left(b_{k}^{2}+b_{k^{\prime}}^{2}\right)^{2} \approx 1$. We see that $P_{\sigma}^{L}(t)$ reaches its first minimum at $\Delta T=\pi / \Delta E$, which is

$$
P_{\sigma}^{L}=b_{k}^{4}+b_{k^{\prime}}^{4}-2 b_{k}^{2} b_{k^{\prime}}^{2}=\left(b_{k}^{2}-b_{k^{\prime}}^{2}\right)^{2} .
$$

As a result, the tunneling probability into the right well is given by $\Delta P=4 b_{k}^{2} b_{k^{\prime}}^{2}$.

To validate our approximation, we select two typical states ( $k=5858$ and 5826) that can be well approximated by two eigenstates, $|k\rangle_{\sigma}$ and $\left|k^{\prime}\right\rangle_{\sigma}$, calculate $b_{k}$ and $b_{k^{\prime}}$, and compare the approximate result Eq. (15) with the accurate result Eq. (5) of the time evolution of $P_{L}^{\sigma}(t)$, as shown in Figs. 9(a) and 9(b). We observe a good agreement.

Both type-I and type-II classes have the cosine time evolution of $P_{\sigma}^{L}$, but they are well separated in the $1 / \Delta T, \Delta P$ plane. The main reason lies in the phase difference between the wave functions on the sublattices $A$ and $B$. Particularly, the phases can have $\pi$ difference, or they can be the same, as illustrated by the eigenwave functions in Figs. 9(a) and 9(b). Due to the phase differences, the energy difference $\Delta E$ (thus the tunneling rate $R$ ) differs as well. For the eigenwave functions with antiphase with respect to $A$ and $B$, the energy difference is considerably smaller than that for the case of identical phases, resulting in the separation exemplified in Fig. 4(a).

For class III pattern, the quantity $|\bar{\psi}(t)\rangle_{k, \sigma}$ can be approximated by three eigenstates,

$$
|\bar{\psi}\rangle_{k, \sigma} \approx b_{k}|k\rangle_{\sigma}+b_{k^{\prime}}\left|k^{\prime}\right\rangle_{\sigma}+b_{k^{\prime \prime}}\left|k^{\prime \prime}\right\rangle_{\sigma}
$$

where $b_{k^{\prime \prime}}$ is comparable to $b_{k^{\prime}}$, and they are smaller than $b_{k}$. Normalization condition requires

$$
b_{k}^{2}+b_{k^{\prime}}^{2}+b_{k^{\prime \prime}}^{2} \approx 1 .
$$

For this class, the states are generally not polarized and they distribute approximately evenly in the left and the right well. We thus have

$$
\langle k \mid k\rangle_{\sigma}^{L} \approx\left\langle k^{\prime} \mid k^{\prime}\right\rangle_{\sigma}^{L} \approx\left\langle k^{\prime \prime} \mid k^{\prime \prime}\right\rangle_{\sigma}^{L} \approx 1 / 2 .
$$

Substituting this relation into Eq. (5), we obtain the time evolution of the left-well probability as

$$
\begin{aligned}
P_{\sigma}^{L}(t) \approx & b_{k}^{2}\langle k \mid k\rangle_{\sigma}^{L}+b_{k^{\prime}}^{2}\left\langle k^{\prime} \mid k^{\prime}\right\rangle_{\sigma}^{L}+b_{k^{\prime \prime}}^{2}\left\langle k^{\prime \prime} \mid k^{\prime \prime}\right\rangle_{\sigma}^{L}+2 b_{k} b_{k^{\prime}}\left\langle k \mid k^{\prime}\right\rangle_{\sigma}^{L} \\
& \times \cos \left(\Delta E^{\prime} \cdot t\right)+2 b_{k} b_{k^{\prime \prime}}\left\langle k \mid k^{\prime \prime}\right\rangle_{\sigma}^{L} \cos \left(\Delta E^{\prime \prime} \cdot t\right) \\
\approx & 1 / 2+2 b_{k} b_{k^{\prime}}\left\langle k \mid k^{\prime}\right\rangle_{\sigma}^{L} \cos \left(\Delta E^{\prime} \cdot t\right) \\
& +2 b_{k} b_{k^{\prime \prime}}\left\langle k \mid k^{\prime \prime}\right\rangle_{\sigma}^{L} \cos \left(\Delta E^{\prime \prime} \cdot t\right),
\end{aligned}
$$

where $\Delta E^{\prime}=E_{k^{\prime}}-E_{k}, \Delta E^{\prime \prime}=E_{k^{\prime \prime}}-E_{k}, b_{k},\left\langle k \mid k^{\prime}\right\rangle_{\sigma}^{L}$, and $\left\langle k \mid k^{\prime \prime}\right\rangle_{\sigma}^{L}$ can be determined numerically. A representative case is shown in Fig. 9(c) with both accurate result in Eq. (5) and the approximation in Eq. (16). It can be seen that the approximated results agree with those from the exact calculation reasonably well. For more complicated cases that have four and more large coefficients of $b_{k}$, the above approximation method is still valid, which has been verified by direct numeric calculations.

\section{CONCLUSION AND DISCUSSION}

A fundamental problem in nonlinear dynamics and quantum physics is the manifestation of classical chaos in quantum systems in the presence of many-body interactions. This issue has been studied but only for nonrelativistic quantum systems [66-69]. We address this problem in relativistic quantum 
mechanics using graphene systems in the setting of resonant tunneling, where the electron-electron Coulomb interactions are described by the mean-field Hubbard Hamiltonian. A resonant tunneling system consists of two symmetric potential wells separated by a potential barrier, and the geometric shape of the whole domain can be chosen to generate integrable or chaotic dynamics in the classical limit. By calculating a large number of eigenenergies and eigenstates, we uncover a class of localized spin-polarized states with near-zero tunneling in the classically integrable systems (e.g., those of rectangular domains). The physical origin of the spin-polarized states can be attributed to the a combined effect of electron-electron interaction, the pseudospin freedom of graphene sublattices, and the potential barrier. Note that the localization phenomenon is somewhat similar to the general localization effect in one-dimensional fermion systems, i.e., the addition of an arbitrary weak barrier at a given point effectively freezes the tunneling through the barrier [88]. However, the underling mechanism in our case is different.

We show that the traditional quantity to characterize quantum tunneling dynamics, namely the tunneling rate, is inadequate for describing the tunneling behavior associated with the localized spin-polarized states. In fact, the tunneling strength is also necessary. For the spin-polarized states, not only can the tunneling rate be negligibly small, but also the tunneling strength. For these states, the spin-up and spin-down electrons are separated in space by the potential barrier. As a result, if an electron occupies a spin-up state, it will remain so for all times. This should be compared with the case of absence of many-body interactions, where the tunneling strength can typically be much larger.

When the geometry is changed so that the classical dynamics becomes chaotic, the spreads in both the tunneling rate and strength are greatly suppressed. The states with extremely small tunneling rate and strength are effectively removed. Study of three representative systems with a chaotic component in the classical limit indicates that a more chaotic system has a stronger ability to regularize the tunneling dynamics. The main message is then that chaos can significantly enhance the tunneling process in realistic situations where electron-electron interactions are present. This implies that classical chaos is capable of facilitating greatly relativistic quantum tunneling, which is desirable in the development of nanoscale devices such as graphene-based resonant-tunneling diodes.

Finally, we wish to discuss and justify the mean-field approach to solving the Hubbard model for graphene systems. First, in Ref. [81], it was indicated that the self-consistent mean-field approach is accurate when the Hubbard interaction strength $U$ is not too large. In our study we used $U=1.2 t$, which is well within the regime of validity of the mean-field Hubbard model, i.e., $U \lesssim 2 t$. Second, in Ref. [83], the dynamical properties of edge state magnetism in graphene systems were investigated. The results of static spin polarization from the mean-field theory were also compared with those from the quantum Monte Carlo (QMC) approach. A main conclusion of Ref. [83] was that the results from the two approaches agree with each other well in terms of the static properties. With respect to dynamic properties, the agreement holds but for narrow graphene ribbons. In general, the Hubbard model is paradigmatic in that it really does capture the electron-electron interactions in graphene systems and the self-consistent mean-field approach is effective to analyze the effects of the interactions. In fact, a recent paper investigated edge spin polarization for large systems, e.g., $10^{4}$ carbon atoms, and it concluded that if the environment time scale $\tau_{\text {env }}$ is much shorter than $\tau_{q d}$, the system is pushed into the same classical Néel-like state again and again. As a result, the state cannot decay, which is known as the quantum Zeno effect [89]. Furthermore, our results do not require perfect spin polarization at opposite zigzag edges. In the presence of quantum fluctuations, if there are noticeable remanent edge magnetic states, which introduce left-right asymmetry to the spin electrons, with the central potential barrier the eigenstates will be spin polarized for the left and the right domains.

\section{ACKNOWLEDGMENTS}

This work was supported by AFOSR under Grant No. FA9550-12-1-0095 and by ONR under Grant No. N0001408-1-0627. L.H. was also supported by NSF of China under Grants No. 11135001 and No. 11375074.
[1] H.-J. Stöckmann, Quantum Chaos: An Introduction (Cambridge University Press, New York, 1999).

[2] F. Haake, Quantum Signatures of Chaos, 3rd ed., Springer series in synergetics (Springer, Berlin, 2010).

[3] O. Bohigas, M. J. Giannoni, and C. Schmit, Phys. Rev. Lett. 52, 1 (1984).

[4] M. V. Berry, Proc. R. Soc. London Ser. A Math. Phys. Eng. Sci. 400, 229 (1985).

[5] M. V. Berry and M. Robnik, J. Phys. A Math. Gen. 19, 649 (1986).

[6] S. W. McDonald and A. N. Kaufman, Phys. Rev. Lett. 42, 1189 (1979).
[7] S. W. McDonald and A. N. Kaufman, Phys. Rev. A 37, 3067 (1988).

[8] E. J. Heller, Phys. Rev. Lett. 53, 1515 (1984).

[9] E. B. Bogomolny, Physica D 31, 169 (1988).

[10] R. L. Waterland, J. M. Yuan, C. C. Martens, R. E. Gillilan, and W. P. Reinhardt, Phys. Rev. Lett. 61, 2733 (1988).

[11] M. V. Berry, Proc. R. Soc. London Ser. A Math. Phys. Eng. Sci. 423, 219 (1989).

[12] B. Eckhardt, G. Hose, and E. Pollak, Phys. Rev. A 39, 3776 (1989).

[13] R. V. Jensen, M. M. Sanders, M. Saraceno, and B. Sundaram, Phys. Rev. Lett. 63, 2771 (1989). 
[14] H. J. Stockmann and J. Stein, Phys. Rev. Lett. 64, 2215 (1990).

[15] B. Eckhardt, J. M. G. Llorente, and E. Pollak, Chem. Phys. Lett. 174, 325 (1990).

[16] R. Blümel, I. H. Davidson, W. P. Reinhardt, H. Lin, and M. Sharnoff, Phys. Rev. A 45, 2641 (1992).

[17] M. Kus, J. Zakrzewski, and K. Zyczkowski, Phys. Rev. A 43, 4244 (1991).

[18] R. V. Jensen, Nature (London) 355, 311 (1992).

[19] R. V. Jensen, Nature (London) 355, 591 (1992).

[20] T. S. Monteiro, D. Delande, and J. P. Connerade, Nature (London) 387, 863 (1997).

[21] C. P. Malta, M. A. M. deAguiar, and A. M. Ozorio deAlmeida, Phys. Rev. A 47, 1625 (1993).

[22] G. G. dePolavieja, F. Borondo, and R. M. Benito, Phys. Rev. Lett. 73, 1613 (1994).

[23] T. M. Fromhold, P. B. Wilkinson, F. W. Sheard, L. Eaves, J. Miao, and G. Edwards, Phys. Rev. Lett. 75, 1142 (1995).

[24] P. Bellomo and T. Uzer, Phys. Rev. A 51, 1669 (1995).

[25] O. Agam, Phys. Rev. B 54, 2607 (1996).

[26] R. Akis, D. K. Ferry, and J. P. Bird, Phys. Rev. Lett. 79, 123 (1997).

[27] F. P. Simonotti, E. Vergini, and M. Saraceno, Phys. Rev. E 56, 3859 (1997).

[28] L. Kaplan and E. J. Heller, Ann. Phys. 264, 171 (1998).

[29] E. E. Narimanov and A. D. Stone, Phys. Rev. Lett. 80, 49 (1998).

[30] L. Kaplan, Nonlinearity 12, R1 (1999).

[31] J. P. Keating and S. D. Prado, Proc. R. Soc. A Math. Phys. Eng. Sci. 457, 1855 (2001).

[32] H. Schanz and T. Kottos, Phys. Rev. Lett. 90, 234101 (2003).

[33] R. Blümel and U. Smilansky, Phys. Rev. Lett. 60, 477 (1988).

[34] R. Blümel and U. Smilansky, Physica D 36, 111 (1989).

[35] R. A. Jalabert, H. U. Baranger, and A. D. Stone, Phys. Rev. Lett. 65, 2442 (1990).

[36] C. M. Marcus, A. J. Rimberg, R. M. Westervelt, P. F. Hopkins, and A. C. Gossard, Phys. Rev. Lett. 69, 506 (1992).

[37] Y.-C. Lai, R. Blümel, E. Ott, and C. Grebogi, Phys. Rev. Lett. 68, 3491 (1992)

[38] R. Ketzmerick, Phys. Rev. B 54, 10841 (1996).

[39] A. S. Sachrajda, R. Ketzmerick, C. Gould, Y. Feng, P. J. Kelly, A. Delage, and Z. Wasilewski, Phys. Rev. Lett. 80, 1948 (1998).

[40] B. Huckestein, R. Ketzmerick, and C. H. Lewenkopf, Phys. Rev. Lett. 84, 5504 (2000).

[41] G. Casati, I. Guarneri, and G. Maspero, Phys. Rev. Lett. 84, 63 (2000).

[42] A. P. S. de Moura, Y.-C. Lai., R. Akis, J. P. Bird, and D. K. Ferry, Phys. Rev. Lett. 88, 236804 (2002).

[43] R. Crook, C. G. Smith, A. C. Graham, I. Farrer, H. E. Beere, and D. A. Ritchie, Phys. Rev. Lett. 91, 246803 (2003).

[44] K. S. Novoselov, A. K. Geim, S. V. Morozov, D. Jiang, Y. Zhang, S. V. Dubonos, I. V. Grigorieva, and A. A. Firsov, Science 306, 666 (2004)

[45] C. Berger, Z. M. Song., T. B. Li., X. B. Li, A. Y. Ogbazghi, R. F. Z. T. Dai, A. N. Marchenkov., E. H. Conrad, P. N. First, and W. A. de Heer, J. Phys. Chem. B 108, 19912 (2004).

[46] K. S. Novoselov, A. K. Geim, S. V. Morozov, D. Jiang, M. I. Katsnelson, I. V. Grigorieva, S. V. Dubonos, and A. A. Firsov, Nature (London) 438, 197 (2005).

[47] Y. B. Zhang, Y. W. Tan, H. L. Stormer, and P. Kim, Nature (London) 438, 201 (2005).
[48] A. H. Castro Neto, F. Guinea, N. M. R. Peres, K. S. Novoselov, and A. K. Geim, Rev. Mod. Phys. 81, 109 (2009).

[49] N. M. R. Peres, Rev. Mod. Phys. 82, 2673 (2010).

[50] S. D. Sarma, S. Adam, E. H. Hwang, and E. Rossi, Rev. Mod. Phys. 83, 407 (2011).

[51] M. V. Berry and R. J. Mondragon, Proc. R. Soc. London Ser. A Math. Phys. Eng. Sci. 412, 53 (1987).

[52] L. Huang, Y.-C. Lai, D. K. Ferry, S. M. Goodnick, and R. Akis, Phys. Rev. Lett. 103, 054101 (2009).

[53] L. Huang, Y.-C. Lai, and C. Grebogi, Phys. Rev. E 81, 055203 (2010).

[54] L. L. Jiang, L. Huang, R. Yang, and Y.-C. Lai, Appl. Phys. Lett. 96, 262114 (2010).

[55] R. Yang, L. Huang, Y.-C. Lai, and C. Grebogi, Europhys. Lett. 94, 40004 (2011).

[56] R. Yang, L. Huang, Y.-C. Lai, and L. M. Pecora, Appl. Phys. Lett. 100, 093105 (2012).

[57] L. Ying, L. Huang, Y.-C. Lai, and C. Grebogi, Phys. Rev. B 85, 245448 (2012).

[58] X. Ni, L. Huang, Y.-C. Lai, and L. M. Pecora, Europhys. Lett. 98, 50007 (2012).

[59] X. Ni, L. Huang, Y.-C. Lai, and C. Grebogi, Phys. Rev. E 86 , 016702 (2012).

[60] H. Y. Xu, L. Huang, Y.-C. Lai, and C. Grebogi, Phys. Rev. Lett. 110, 064102 (2013).

[61] L. Huang, R. Yang, Y.-C. Lai, and D. K. Ferry, J. Phys. Condens. Matter 25, 085502 (2013).

[62] L. Ying, L. Huang, Y.-C. Lai, and Y. Zhang, J. Phys. Condens. Matter 25, 105802 (2013).

[63] R. Yang, L. Huang, Y.-C. Lai, C. Grebogi, and L. M. Pecora, Chaos 23, 013125 (2013).

[64] G. L. Wang, L. Ying, Y.-C. Lai, and C. Grebogi, Phys. Rev. E 87, 052908 (2013).

[65] X. Ni, L. Huang, L. Ying, and Y.-C. Lai, Phys. Rev. B 87, 224304 (2013).

[66] K.-H. Ahn, K. Richter, and I.-H. Lee, Phys. Rev. Lett. 83, 4144 (1999).

[67] R. Shankar, Rev. Mod. Phys. 80, 379 (2008).

[68] D. Ullmo, Rep. Prog. Phys. 71, 026001 (2008).

[69] J. M. G. Gómez, K. Kar, V. K. B. Kota, R. A. Molina, A. Relaño, and J. Retamosa, Phys. Rep. 499, 103 (2011).

[70] J. Hubbard, Proc. R. Soc. London Ser. A Math. Phys. Sci. 276, 238 (1963).

[71] F. H. L. Essler, The One-Dimensional Hubbard Model (Cambridge University Press, Cambridge, UK, 2005).

[72] A. Altland and B. Simons, Condensed Matter Field Theory, 2nd ed. (Cambridge University Press, New York, 2010).

[73] L. M. Pecora, H. Lee, D. H. Wu, T. Antonsen, M. J. Lee, and E. Ott, Phys. Rev. E 83, 065201 (2011).

[74] L. A. Bunimovich, Chaos 11, 802 (2001).

[75] O. V. Yazyev, Rep. Prog. Phys. 73, 056501 (2010).

[76] M. Fujita, K. Wakabayashi, K. Nakada, and K. Kusakabe, J. Phys. Soc. Jpn. 65, 1920 (1996).

[77] J. Fernandez-Rossier and J. J. Palacios, Phys. Rev. Lett. 99, 177204 (2007).

[78] F. Liu, Y. L. Li, X. Y. Liu, and J. F. Kang, Appl. Phys. Lett. 99, 243503 (2011).

[79] Y. C. Chang and S. Haas, Phys. Rev. B 83, 085406 (2011).

[80] Y. Hancock, A. Uppstu, K. Saloriutta, A. Harju, and M. J. Puska, Phys. Rev. B 81, 245402 (2010). 
[81] H. Feldner, Z. Y. Meng, A. Honecker, D. Cabra, S. Wessel, and F. F. Assaad, Phys. Rev. B 81, 115416 (2010).

[82] K. Wakabayashi, M. Sigrist, and M. Fujita, J. Phys. Soc. Jpn. 67, 2089 (1998).

[83] H. Feldner, Z. Y. Meng, T. C. Lang, F. F. Assaad, S. Wessel, and A. Honecker, Phys. Rev. Lett. 106, 226401 (2011).

[84] J. Martin, N. Akerman, G. Ulbricht, T. Lohmann, J. H. Smet, K. V. Klitzing, and A. Yacoby, Nat. Phys. 4, 144 (2008).
[85] T. Ando, J. Phys. Soc. Jpn. 74, 777 (2005).

[86] L. Brey and H. A. Fertig, Phys. Rev. B 73, 235411 (2006).

[87] M. Drienovsky, F.-X. Schrettenbrunner, A. Sandner, D. Weiss, J. Eroms, M.-H. Liu, F. Tkatschenko, and K. Richter, Phys. Rev. B 89, 115421 (2014).

[88] C. L. Kane and M. P. A. Fisher, Phys. Rev. B 46, 15233 (1992).

[89] M. Golor, S. Wessel, and M. J. Schmidt, Phys. Rev. Lett. 112, 046601 (2014). 